\title{
Identificación de áreas de recarga a través de un modelo hidrológico en la región del Arco Seco de Panamá
}

\author{
Noel Amaya, Job Osvaldo \\ Centro de Investigaciones Hidráulicas e Hidrotécnicas de la Universidad Tecnológica de Panamá. \\ Panamá, Panamá \\ job.noel@utp.ac.pa \\ Fábrega, José \\ Centro de Investigaciones Hidráulicas e Hidrotécnicas de la Universidad Tecnológica de Panamá. \\ Panamá, Panamá \\ jose.fabrega@utp.ac.pa \\ Casstrellón, María Gabriela \\ Asociada al Centro de Investigaciones Hidráulicas e Hidrotécnicas de la Universidad Tecnológica de \\ Panamá. \\ Panamá, Panamá \\ mariag.castrellon@gmail.com
}

\section{Abstract}

Variations of the hydrological cycle in the groundwater recharge mechanisms have few studies/researches in tropical countries such as Panama. In recent decades, regions of the country such as the Dry Arc, have faced droughts and supply issues mainly due to anthropogenic alterations and the effects of climate change. Likewise, the groundwater recharge areas are not well defined and there has been a disorderly exploitation of groundwater, producing an increase in withdrawals, affecting their quality and quantity. This research aims to understand the interactions of hydrometeorological variables through the application of the SWAT+ hydrological model to identify recharge areas in the La Villa river basin. Generic meteorological data, and information from the Basin are combined to generate Hydrological Response Units to provide a spatial and physical analysis of recharge mechanisms in the region. Results show that most of the recharge areas in the study area are changing their land use, using it to a greater extent for agriculture and livestock. These activities have an impact on forest cover and significantly alter the infiltration capacity of the soil and, therefore, affecting the groundwater recharge. Through this research, the aim is to promote the management of water resources and land use policies to guarantee a 
sustainable use of groundwater recharge zones in the region.

Keywords: Hydrology, SWAT+, Recharge Areas, Hydrological Response Units, Groundwater.

\section{Resumen}

Las variaciones del ciclo hidrológico en los mecanismos de recarga de las aguas subterráneas están poco estudiadas en los países tropicales como Panamá. En las últimas décadas, regiones del país como el Arco Seco, han enfrentado sequías y problemas de suministro principalmente debido a alteraciones antropogénicas y efectos del cambio climático. Igualmente, las áreas de recarga de aguas subterráneas no están bien definidas y ha existido una explotación desordenada de las aguas subterráneas produciendo un aumento de extracciones, afectando su calidad y cantidad. Esta investigación tiene como objetivo comprender las interacciones de variables hidrometeorológicas a través de la aplicación del modelo hidrológico SWAT+ para identificar áreas de recarga en la cuenca del río La Villa. Datos meteorológicos genéricos, e información de la Cuenca se combinan para generar Unidades de Respuesta Hidrológica para proporcionar un análisis espacial y físico de los mecanismos de recarga en la región. Los resultados presentan que la mayoría de las áreas de recarga en la zona de estudio están cambiando su uso de suelo, utilizándolo en mayor medida para la agricultura y la ganadería. Estas actividades tienen un impacto en la cubierta forestal y alteran significativamente la capacidad de infiltración del suelo y, por lo tanto, afectan la recarga de aguas subterráneas. A través de esta investigación se busca potenciar la gestión de los recursos hídricos y las políticas de uso de la tierra para garantizar un uso sostenible de las zonas de recarga de aguas subterráneas en la región.

Palabras claves: Hidrología, SWAT+, Áreas de recarga, Unidades de Respuesta Hidrológica, Aguas Subterráneas.

\section{INTRODUCCIÓN}

Las variaciones del ciclo hidrológico en los mecanismos de recarga de las aguas subterráneas están poco estudiadas en los países tropicales como Panamá. Panamá, recibe una precipitación promedio anual de $2.924 \mathrm{~mm}$ y tiene 119.5 mil millones de $\mathrm{m} 3$ de agua disponible por año (Consejo Nacional del Agua, 2016). Debido a esta abundancia, el agua superficial ha sido tradicionalmente la fuente para el consumo humano y la agricultura. Sin embargo, en las últimas décadas, algunas regiones del país han enfrentado sequías y problemas de suministro debido principalmente a alteraciones antropogénicas y efectos 
del cambio climático (Ministerio de Ambiente, 2019).

La región del Arco Seco en Panamá, localizada principalmente en la península de Azuero, en las Ilanuras y colinas costeras orientales de las provincias de Los Santos y Herrera, y parte del litoral Sur de la provincia de Coclé, es un claro ejemplo de esta situación (Autoridad Nacional del Ambiente, 2013). La precipitación en esta región es impulsada principalmente por la migración latitudinal anual de la Zona de Convergencia Intertropical, lo que resulta en el $95 \%$ de la precipitación anual en la temporada de lluvias (mayo-diciembre), y el 5\% restante durante la temporada seca (enero-abril). Esta distribución anual de la precipitación causa escasez de agua superficial durante la estación seca. Por otro lado, las áreas de recarga de aguas subterráneas en esta región no están bien definidas y ha existido una explotación desordenada de las aguas subterráneas, produciendo un aumento de extracciones con un incremento del 75\% entre los años 2002 a 2010, extrayendo aproximadamente 700,000 m3/día (Autoridad Nacional del Ambiente, 2013). Estas circunstancias afectan tanto a la calidad (salinización en zonas costeras y contaminación de pozos por productos químicos) y cantidad (bombeo más de lo necesario del agua empleada). Este trabajo enfocado en la región del Arco Seco, en la cuenca del río La Villa, tiene como objetivo comprender mejor las interacciones entre la precipitación, el agua superficial y el agua subterránea, a través de la aplicación de un modelo hidrológico para identificar áreas de recarga en una cuenca hidrográfica en la región del Arco Seco de Panamá.

\section{MÉTODO}

\section{A. Área de Estudio}

El sitio de estudio es la cuenca del río La Villa, ubicada en la península de Azuero, en la región del Arco Seco Centroamericano (Ver Fig. 1). Es la Cuenca \#128 y comprende las provincias de Herrera y Los Santos, posee un área de drenaje de $1284.3 \mathrm{~km}^{2}$, y la longitud de su río principal es de $117 \mathrm{~km}$ (ETESA, 2008), (Cornejo et al., 2017). La elevación media de la cuenca es de $135 \mathrm{msnm}$ y cuenta con una precipitación media anual de $1868 \mathrm{~mm}$ con sequías marcadas durante la época seca (ETESA, 2008). Esta cuenca es de gran importancia para la región ya que abastece de agua para consumo humano y usos domésticos a más de 94,000 personas.

\section{B. Modelo Hidrológico}

El manejo de la data del modelo hidrológico se llevó a cabo a través del programa Quantum GIS, que es un software de código libre que permite manejar formatos ráster y vectoriales, donde se insertaron diferentes tipos de capas (Modelo de Elevación Digital 
30m; Cobertura Boscosa, y el mapa de uso de suelos para Panamá de la FAO). Luego esta información fue procesada a través del software "Soil \& Water Assessment Tool (SWAT+)", que es un modelo que se utiliza para representar interacciones y procesos que ocurren dentro de una cuenca hidrológica, como cuantificar caudales y volúmenes de aguas subterráneas, así como también para predecir impactos ambientales en el uso de suelo, prácticas de manejo de suelos y cambio climático.

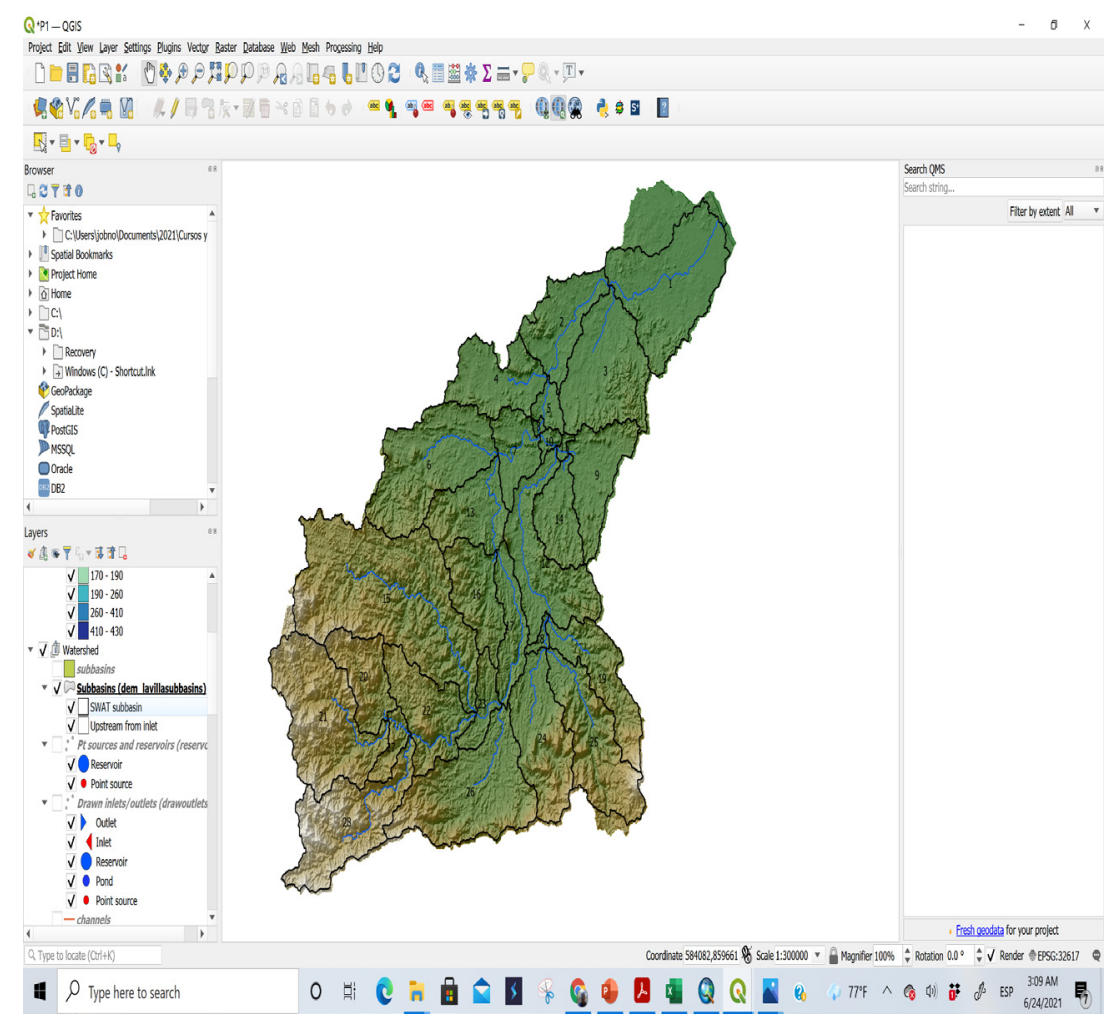

Fig. 1. Cuenca Hidrográfica del río La Villa. Fuente: Creación propia.

Adicionalmente, para nuestro análisis se utilizó el generador de data climática del modelo SWAT+ para la obtención de variables climáticas como la temperatura, y la radiación solar, así como también se utilizaron estaciones puntuales de precipitación estimadas a través del "Climate Forecast System Reanalysis".

Posteriormente, con toda la información necesaria en el modelo se crearon 276 Unidades de Respuesta Hidrológica (HRUs por sus siglas en inglés), y la corrida del modelo se llevó a cabo por un periodo de 24 años (1/1/1987 - 1/1/2011), incluyendo 3 años de corrida del modelo sin almacenamiento de información, con la finalidad de generar resultados basados en los promedios anuales de infiltración que drenan de la capa del suelo hacia la zona vadosa. 


\section{RESULTADOS}

En esta primera corrida del modelo, utilizando información espacial y no local, se obtuvieron los resultados preliminares del modelo de identificación de potenciales zonas de recarga en la cuenca del río La Villa. La figura 2, muestra un mapa de la cuenca del río La Villa, donde producto de la corrida del modelo, identificó que las zonas con un mayor índice de infiltración en la cuenca del río La Villa se presentan en las partes altas de la Cuenca, sobre todo con mayor ubicación en los distritos de Las Minas y Los Pozos donde hay áreas con porcentajes de cobertura de bosques maduros y secundarios, y bosques intervenidos.

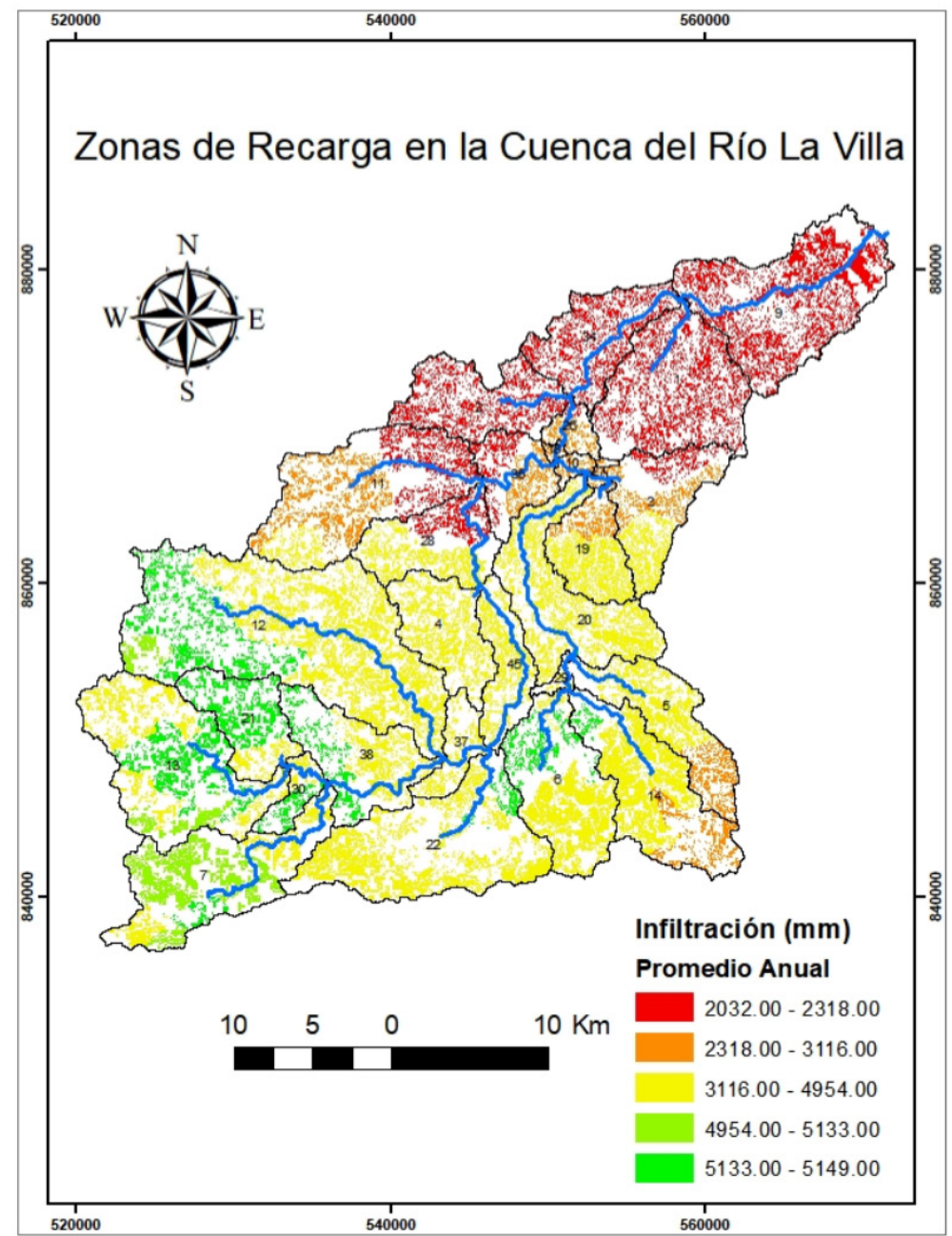

Fig. 2. Infiltración promedio anual en la cuenca del río La Villa. 


\section{CONCLUSIONES}

Con la finalidad de comprender mejor los procesos hidrológicos dominantes y proporcionar una comprensión espacial y física de los mecanismos de recarga en la región, la mayoría de las áreas de recarga en la región del Arco Seco han cambiado o están cambiando su uso de suelo, utilizándolo en mayor medida para la agricultura y la ganadería. Estas actividades tienen un impacto en la cubierta forestal y alteran significativamente la capacidad de infiltración del suelo y, por lo tanto, afectan la recarga de aguas subterráneas. Se requiere tomar acciones ante estos impactos que están afectando a mediano y largo plazo las fuentes de agua de la población. A pesar de los altos valores producidos en esta primera corrida preliminar del modelo, se ha obtenido una primera visualización y análisis de la situación actual con las zonas de recarga. En los siguientes pasos, se correrá el modelo con información recabada de campo para tener valores de infiltración más precisos y poder utilizar los resultados de este modelo hidrológico para informar y concientizar la gestión de los recursos hídricos y mejorar las políticas de uso de la tierra para garantizar un uso sostenible de las zonas de recarga de aguas subterráneas en la región del Arco Seco de Panamá.

\section{Referencias}

[1] Ministerio de Ambiente, "Estrategia Nacional de Cambio Climático, 2050," ISBN: 978-9962-8511-27, 2019.

[2] Autoridad Nacional del Ambiente, "Las aguas subterráneas de la región del Arco Seco y la importancia de su conservación," ISBN 978-9962-651-80-2, 2013.

[3] Consejo Nacional del Agua, "Plan Nacional de Seguridad Hídrica 2015-2050: Agua para Todos," Panamá, República de Panamá, ISBN: 978-9962-5581-0-1, 2016.

[4] Cornejo, A., E. López-López, R. A., Ruiz-Picos, J. E. Sedeño-Díaz, B. Armitage, T. Arefina, C. Nieto, A. Tuñón, M. Molinar, T. Ábrego, E. Pérez, A.R., Tuñón, J. Magué, A. Rodríguez, J. Pineda, J. Cubilla \& I. M. Avila Quintero. "Diagnóstico de la condición ambiental de los afluentes superficiales de Panamá," 326 p, 2017.

\section{Autorización y Licencia CC}

Los autores autorizan a APANAC XVIII a publicar el artículo en las actas de la conferencia en Acceso Abierto (Open Access) en diversos formatos digitales (PDF, HTML, EPUB) e integrarlos en diversas plataformas online como repositorios y bases de datos bajo la licencia CC:

Attribution-NonCommercial-ShareAlike 4.0 International (CC BY-NC-SA 4.0) https://creativecommons.org/ licenses/by-nc-sa/4.0/.

Ni APANAC XVIII ni los editores son responsables ni del contenido ni de las implicaciones de lo expresado en el artículo. 